Accurate and efficient radiation transport in optically thick media -- by means of the Symbolic Implicit Monte Carlo method in the difference formulation

A. Szoke, E. D. Brooks, M.S. McKinley, F. Daffin

March 31, 2005

Computational Methods in Transport Workshop Lake Tahoe, CA, United States September 11, 2004 through September 16, 2004 
This document was prepared as an account of work sponsored by an agency of the United States Government. Neither the United States Government nor the University of California nor any of their employees, makes any warranty, express or implied, or assumes any legal liability or responsibility for the accuracy, completeness, or usefulness of any information, apparatus, product, or process disclosed, or represents that its use would not infringe privately owned rights. Reference herein to any specific commercial product, process, or service by trade name, trademark, manufacturer, or otherwise, does not necessarily constitute or imply its endorsement, recommendation, or favoring by the United States Government or the University of California. The views and opinions of authors expressed herein do not necessarily state or reflect those of the United States Government or the University of California, and shall not be used for advertising or product endorsement purposes. 


\title{
Accurate and efficient radiation transport in optically thick media - by means of the Symbolic Implicit Monte Carlo method in the difference formulation *
}

\author{
Abraham Szőke, Eugene D. Brooks III, \\ Michael Scott McKinley, and Frank C. Daffin \\ University of California, Lawrence Livermore National Laboratory, \\ P.O. Box 808, Livermore, CA 94550, USA \\ szoke1@llnl.gov, brooks3@llnl.gov, \\ mckinley9@llnl.gov,daffin1@llnl.gov
}

The equations of radiation transport for thermal photons are notoriously difficult to solve in thick media without resorting to asymptotic approximations such as the diffusion limit. One source of this difficulty is that in thick, absorbing media thermal emission is almost completely balanced by strong absorption. In a previous publication [SB03], the photon transport equation was written in terms of the deviation of the specific intensity from the local equilibrium field. We called the new form of the equations the difference formulation. The difference formulation is rigorously equivalent to the original transport equation. It is particularly advantageous in thick media, where the radiation field approaches local equilibrium and the deviations from the Planck distribution are small. The difference formulation for photon transport also clarifies the diffusion limit. In this paper, the transport equation is solved by the Symbolic Implicit Monte Carlo (SIMC) method and a comparison is made between the standard formulation and the difference formulation. The SIMC method is easily adapted to the derivative source terms of the difference formulation, and a remarkable reduction in noise is obtained when the difference formulation is applied to problems involving thick media.

\section{Introduction}

The transport of thermal photons in thick media is of sufficient importance that substantial effort has been expended in developing both deterministic

\footnotetext{
* Work performed under the auspices of the U. S. Department of Energy by the Lawrence Livermore National Laboratory under contract No. W-7405-ENG-48.
} 
[Mih78] and Monte Carlo [FC71] methods for its solution. The difficulties associated with thick media have been severe enough to necessitate solving asymptotic approximations, such as the Eddington and diffusion approximations [PB83], instead of solving the full transport equation.

Asymptotic methods do give the right solution to the transport equation in uniformly thick media, like stellar interiors. Nevertheless, in many problems of interest the medium is a mixture of thick and thin regions; moreover, some regions of interest may be thin for some radiation frequencies and thick for others.

Although a lot of progress has been made in numerical calculations for such complicated systems using asymptotic methods, they suffer from several defects. One of them is an unphysical energy propagation rate when the method is applied outside its proper domain, e.g. to optically thin regions. This led to the development of ad hoc corrections such as flux limiters [Pom82]. Another defect is that asymptotic methods are unable to satisfy correct boundary conditions. Time honored "fixes" are the Marshak [Mrs47] and Mark [Mrk47] boundary conditions, but these incorrect boundary conditions distort ubiquitous boundary layers. More importantly, it is difficult to estimate or measure the errors incurred by the approximations. Only an accurate solution of the transport equation is able to eliminate the above defects.

Several hurdles have stood in the way of producing accurate Monte Carlo solutions of the transport equation in thick media. The first one was overcome by the development of a Monte Carlo technique that is numerically stable and provides correct treatment of the stiff coupling between the radiation and the material in the thick limit. Several authors have shown that the radiation matter coupling is properly treated in the Symbolic Implicit Monte Carlo method [Bro86, Nka91], producing a correct implicit solution of the radiation field and the material temperature at the end of a time step [DL04], while effective scattering techniques [FC71, CF73] possess a significant deficiency in this regard.

A second hurdle has been the very significant noise problem, or the equivalent problem of computational efficiency, when Monte Carlo methods are pressed into service for thick systems. The energy is emitted in a zone uniformly, but only particles born within a few mean free paths of a zone boundary have any chance of contributing to the flux across the boundary. Most of the emitted particles are absorbed within the same zone and serve only to compute the equilibrium values of the radiation intensity and temperature in that zone. This situation for the Monte Carlo method, as applied to thermal photon transport, has been a source of frustration for a long time. The local equilibirium value of the radiation intensity in the thick limit is, of course, the black body field for the given local temperature. One would prefer not to waste a lot of processing power computing it.

In an earlier work [SB03], a new formulation was proposed for the transport of thermal photons, referred to as the difference formulation. The main considerations these papers will now be repeated. The natural way of deriv- 
ing the transport equation is to follow the propagation of narrow beams of photons as they are emitted, propagate in vacuo, are scattered and, finally, are absorbed by matter [Cas00, MM84]. In transparent media the absorption, emission and scattering of photons is weak and the transport equation describes the overall propagation very well. Mathematically, the equations of propagation are hyperbolic partial differential equations and their numerical solution is relatively easy and stable in optically thin media.

In optically thick media, however, the probability that a photon propagates in a straight line, unhindered, is very small; radiation transport is dominated by a large number of scattering, absorption and re-emission events. As a result, the solution of the transport equation in thick media is not straightforward. An important example is hot, dense matter with a high absorption coefficient. It results in conditions of local thermodynamic equilibrium (LTE) and very strong emission of photons. The emitted photons, in turn, are quickly reabsorbed, maintaining the temperature of the medium. The net emission (or absorption) is then a small difference between two large terms. The process leads to stiffness of the transport equation: the material and radiation temperature come into equilibrium much faster than any excess energy is transported away. In any numerical method that uses explicit differencing to balance thermal emission with absorption, the stiffness can cause instability, as well as a significant increase in noise for Monte Carlo methods. If the scattering coefficient is high a photon does not propagate in a straight path. This poses a difficulty for methods that are highly dependent upon efficient streaming of photons.

The transformation to the difference formulation, proposed in [SB03], is achieved by considering the difference between the radiation field and the local equilibrium field at each point in the problem domain. The local equilibrium field is a function of the matter temperature, and therefore a function of both space and time. It results in a transport equation that contains only quantities that are small when the system is thick. In particular, the large emission term and its (almost) compensating absorption term are replaced by a pure absorption term for the "difference field". The only sources for the difference field come from the variation of the material temperature in space and time.

Why is the difference formulation interesting? To summarize, the equations are written in terms of quantities that are "natural" in thick media. (The traditional formulation is written in terms of variables that are natural in thin media.) In hot, dense matter the terms describing the nearly equal emission and absorption of photons are eliminated and only the small, net transport terms appear in the equation. We expect that this change of variables will aid in its numerical solution: it will make it less stiff, more numerically stable, and it will reduce the noise in Monte Carlo methods. In fact, preliminary results shown here confirm our expectations. Derivation of the diffusive behavior of the transport equation in thick media is simplified and clarified by the difference formulation. As the difference equation is able to satisfy the correct 
physical boundary conditions, we hope to find a fast and accurate alternative to the radiation diffusion equation. Finally, the new formalism might lead to the development of new numerical methods.

\section{Radiation transport in LTE}

The radiation transport equations will be written down in both the traditional and the difference formulation - in order to introduce the notation and for completeness.

\subsection{Traditional formulation}

Radiation transport and its coupling to matter is described by the equations of radiation hydrodynamics. In their general form, they consist of the equations of hydrodynamics coupled to those of radiation transport and to the interaction of radiation with matter. Excellent treatises have been written by Pomraning [Pom73], Mihalas [MM84] and Castor [Cas00].

In this paper we deal only with a subset of those equations. They are the radiation transport equation, the material energy balance equation and the conservation equation for the sum of the radiation and material energy. Furthermore we assume local thermodynamic equilibrium (LTE) - i.e. that the material has a well defined temperature - it emits radiation thermally. We also assume that the material is at rest or that it moves with constant velocity. In real hydrodynamic cases, where different parts of the material move at different velocities, the "co-moving frame transformation" has to be used and proper account has to be given to kinetic energy and hydrodynamic work [Cas00]. When the local acceleration of the material is significant, general relativity has to be invoked [MA00]. Our equations are written in the rest frame of the material, assumed to be an inertial frame. Otherwise, the scattering terms would have a more complicated angle and frequency dependence.

The transport equation describes the propagation of the radiation field in terms of the specific intensity, $I(\mathbf{x}, t ; \nu, \boldsymbol{\Omega})$, where $\mathbf{x}, t$ are the space and time variables, $\nu$ is the radiation frequency and $\boldsymbol{\Omega}$ is a unit vector in the direction of propagation.

$$
\begin{aligned}
\frac{1}{c} \frac{\partial I(\mathbf{x}, t ; \nu, \boldsymbol{\Omega})}{\partial t}+ & \boldsymbol{\Omega} \cdot \nabla I(\mathbf{x}, t ; \nu, \boldsymbol{\Omega})= \\
& \sigma_{a}^{\prime}(\nu, T(\mathbf{x}, t))[B(\nu, T(\mathbf{x}, t))-I(\mathbf{x}, t ; \nu, \boldsymbol{\Omega})]+Q(I)
\end{aligned}
$$

$B(\nu, T)$ is the thermal (Planck) distribution at the material temperature, $T(\mathbf{x}, t)$, and $c$ is the speed of light. The absorption coefficient, $\sigma_{a}^{\prime}$, and the scattering term, $Q(I)$, will be defined below. The specific intensity is related to the photon distribution function $f(\mathbf{x}, t ; \nu, \boldsymbol{\Omega})$ by

$$
I(\mathbf{x}, t ; \nu, \boldsymbol{\Omega})=\operatorname{ch} \nu f(\mathbf{x}, t ; \nu, \boldsymbol{\Omega})
$$


where $h \nu$ is the photon energy.

In Eq. (1), all the variables, $I, \sigma_{a}^{\prime}, B$ are functions of the independent variables, $\mathbf{x}, t ; \nu, \boldsymbol{\Omega}$ and/or $T(\mathbf{x}, t)$. In the following, the independent variables will mostly be suppressed.

The emission function and the absorption cross sections, corrected for stimulated emission, are

$$
\begin{gathered}
B(\nu, T)=\frac{2 h \nu^{3}}{c^{2}}\left(e^{h \nu / k T}-1\right)^{-1}, \\
\sigma_{a}^{\prime}(\nu, T)=\sigma_{a}(\nu, T)\left(1-e^{-h \nu / k T}\right),
\end{gathered}
$$

with $\sigma_{a}$ being the "ordinary" absorption coefficient, per unit distance.

The scattering terms are denoted by $Q(I)$

$$
\begin{aligned}
Q(I) & =\int_{0}^{\infty} d \nu^{\prime} \int_{4 \pi} d \boldsymbol{\Omega}^{\prime} \frac{\nu}{\nu^{\prime}} \sigma_{s}\left(\nu^{\prime} \rightarrow \nu, \boldsymbol{\Omega} \cdot \boldsymbol{\Omega}^{\prime}\right) I\left(\nu^{\prime}, \boldsymbol{\Omega}^{\prime}\right)\left[1+\frac{c^{2} I(\nu, \boldsymbol{\Omega})}{2 h \nu^{3}}\right] \\
& -\int_{0}^{\infty} d \nu^{\prime} \int_{4 \pi} d \boldsymbol{\Omega}^{\prime} \sigma_{s}\left(\nu \rightarrow \nu^{\prime}, \boldsymbol{\Omega} \cdot \boldsymbol{\Omega}^{\prime}\right) I(\nu, \boldsymbol{\Omega})\left[1+\frac{c^{2} I\left(\nu^{\prime}, \boldsymbol{\Omega}^{\prime}\right)}{2 h \nu^{\prime 3}}\right],
\end{aligned}
$$

where the $\mathbf{x}, t ; T$ dependence of $\sigma_{s}$ has been suppressed. In LTE there are thermodynamic relations among the partial scattering cross sections in Eq. (5). These follow from the observation that, in complete thermal equilibrium, the radiation field reduces to the black body spectrum no matter what the scattering cross sections are. See Eq. (30) below.

The zeroth moment of the intensity gives the radiation energy density

$$
E_{\text {rad }}=\frac{1}{c} \int_{0}^{\infty} d \nu \int_{4 \pi} d \Omega I
$$

and its first moment is the radiation flux vector

$$
\mathbf{F}_{\text {rad }}=\int_{0}^{\infty} d \nu \int_{4 \pi} d \boldsymbol{\Omega} \boldsymbol{\Omega} I
$$

Interaction of radiation with matter is expressed by the conservation law

$$
\frac{\partial E_{m a t}}{\partial t}=\int_{0}^{\infty} d \nu \int_{4 \pi} d \boldsymbol{\Omega} \sigma_{a}^{\prime}[I-B(\nu, T)]-\int_{0}^{\infty} d \nu \int_{4 \pi} d \boldsymbol{\Omega} Q(I)+G,
$$

where $E_{m a t}$ is the energy per unit volume of the material and $G$ is a volume source of energy.

In the absence of hydrodynamic work terms or thermal conductivity, the total energy of the radiation field and the material are conserved

$$
\frac{\partial\left(E_{\text {mat }}+E_{\text {rad }}\right)}{\partial t}+\nabla \cdot \mathbf{F}_{\text {rad }}=G
$$




\subsection{Thick media}

We all have a common-sense concept of a thick medium; we attempt to clarify it here. One property of radiation in thick media is that its distribution is almost isotropic. Another property of thick media is that the transport of energy by radiation is severely hindered.

In the spirit of the first property we define a streaming parameter, $\epsilon_{\text {stream }}$; it is the ratio of the magnitude of the actual radiation flux, $\left|\mathbf{F}_{\text {rad }}\right|$, to the maximum possible one

$$
\epsilon_{\text {stream }}:=\frac{\left|\mathbf{F}_{\text {rad }}\right|}{c E_{\text {rad }}} .
$$

It is clear that $0 \leq \epsilon_{\text {stream }} \leq 1$ and that $\epsilon_{\text {stream }}=1$ only if the radiation streams in one well-defined direction. In thick media, $\epsilon_{\text {stream }}$ is a small parameter: $\epsilon_{\text {stream }} \ll 1$.

In the spirit of the second property, we look at the ratio of the photon mean free path, $l_{\text {rad }}$ and some scale length, $L$. The scale length defines the distance of significant variation in the properties of the material. We define

$$
\epsilon_{\text {space }}:=\frac{4}{3} \frac{l_{\text {rad }}}{L} \text {. }
$$

In thick media $\epsilon_{\text {space }} \ll 1$.

In thick media that strongly absorbs and emits radiation, far from any boundary layer, the diffusion approximation is valid. In the diffusion limit, the photon mean free path is determined by the Rosseland mean opacity, $l_{\text {rad }}=1 / \sigma_{R}$ and the scale length is set by the rate of change in the temperature: $1 / L=\left(1 / 4 T^{4}\right)\left|\nabla\left(T^{4}\right)\right|$. In the diffusive regime the radiation energy density is that of a black body, $E_{r a d}=a T^{4}$ and the diffusion flux is $\mathbf{F}_{\text {rad }}=-\left(a c / 3 \sigma_{R}\right) \nabla\left(T^{4}\right)$. (Both of the preceding formulas are valid to first order in the small parameter $\epsilon_{\text {space }}$.) Simple algebra shows that in the interior of a thick, strongly absorbing and emitting region, without scattering, the two approaches give the same result

$$
\epsilon_{\text {space }} \approx \epsilon_{\text {stream }} .
$$

The time rate of change of conditions in thick media can be estimated in a similar manner. We define a small parameter that is the ratio of the free flight time of a photon to the time rate of change of the temperature

$$
\epsilon_{\text {time }}:=\frac{1}{c \sigma_{R}} \frac{1}{T^{4}} \frac{\partial T^{4}}{\partial t} .
$$

Heating of the material results from radiation transport. Using the smallness of the energy flux, $\epsilon_{\text {stream }} \ll 1$, from the energy balance in a small volume we get the estimate

$$
\epsilon_{\text {time }} \approx \epsilon_{\text {space }}^{2} \frac{3 E_{\text {rad }}}{E_{\text {rad }}+\partial E_{\text {mat }} / \partial\left(T^{4}\right)} .
$$

The parameters $\epsilon_{\text {stream }}, \epsilon_{\text {space }}$ and $\epsilon_{\text {time }}$ are not small in some boundary layers and in the leading edge of thermal waves. 


\section{The difference formulation}

In the introduction we discussed the difficulties of solving the transport equation, Eq. (1), in thick media. In the previous section we identified the streaming parameter, $\epsilon_{\text {stream }}$, that is small in thick media. We now show a very simple exact transformation of the transport equation so that it is written in terms of variables that are small in thick media. We call the result of the transformation the "difference formulation." In the following we show the transformation in a simple case and discuss its remarkable properties.

\subsection{The difference formulation without scattering}

We start by repeating the transport equation, Eq. (1), without scattering

$$
\begin{aligned}
\frac{1}{c} \frac{\partial I(\mathbf{x}, t ; \nu, \boldsymbol{\Omega})}{\partial t} & +\mathbf{\Omega} \cdot \nabla I(\mathbf{x}, t ; \nu, \boldsymbol{\Omega})= \\
& -\sigma_{a}^{\prime}(\nu, T(\mathbf{x}, t))[I(\mathbf{x}, t ; \nu, \boldsymbol{\Omega})-B(\nu, T(\mathbf{x}, t))] .
\end{aligned}
$$

The equation is written in terms of the specific intensity carried by photons, $I(\mathbf{x}, t ; \nu, \mathbf{\Omega})$. The left-hand side of the equation describes their unhindered propagation, the first term on the right-hand side describes their attenuation. The two terms on the left-hand side and the first term on the right-hand side constitute a homogeneous equation. The last term on the right-hand side, $\sigma_{a}^{\prime} B$, is a "source term" that makes the full equation inhomogeneous. It describes the emission of radiation by matter. From our considerations in the previous section, we expect that the difference between the two terms on the right-hand side is of the order of $\epsilon_{\text {stream }}$ in thick media, even though each term by itself is of the relative order unity.

We introduce now a "difference intensity"

$$
D(\mathbf{x}, t ; \nu, \mathbf{\Omega}):=I(\mathbf{x}, t ; \nu, \mathbf{\Omega})-B(\nu, T(\mathbf{x}, t))
$$

and subtract $(1 / c)(\partial B / \partial t)+\boldsymbol{\Omega} \cdot \nabla B$ form both sides of Eq. (15).

$$
\begin{array}{r}
\frac{1}{c} \frac{\partial D(\mathbf{x}, t ; \nu, \boldsymbol{\Omega})}{\partial t}+\mathbf{\Omega} \cdot \nabla D(\mathbf{x}, t ; \nu, \boldsymbol{\Omega})=-\sigma_{a}^{\prime}(\nu, T(\mathbf{x}, t)) D(\mathbf{x}, t ; \nu, \mathbf{\Omega}) \\
-\frac{1}{c} \frac{\partial B(\nu, T(\mathbf{x}, t))}{\partial t}-\boldsymbol{\Omega} \cdot \nabla B(\nu, T(\mathbf{x}, t))
\end{array}
$$

Let us rewrite it with the independent variables suppressed for clarity.

$$
\frac{1}{c} \frac{\partial D}{\partial t}+\boldsymbol{\Omega} \cdot \nabla D=-\sigma_{a}^{\prime} D-\frac{1}{c} \frac{\partial B}{\partial t}-\boldsymbol{\Omega} \cdot \nabla B
$$

It should be emphasized that Eq. (18) and Eq. (15) are completely equivalent. In particular, they are able to satisfy equivalent initial and boundary conditions. The positivity constraint, $I \geq 0$, translates into $D \geq-B$. 
It is important to investigate the properties of the new equation, (18), comparing it to its traditional counterpart, Eq. (15) and, by extension, to Eq. (1). The terms on the left-hand side and the first term on the right-hand side of Eq. (18) are completely analogous to those in Eq. (15): they describe the straight line propagation and the attenuation of the difference intensity, $D$. We conclude that the intensity $I$ and the difference intensity $D$ propagate the same way. In particular, their Green's functions (propagators) are the same.

In contrast, the inhomogeneous source terms have been changed drastically. The source term in Eq. (15) is $\sigma_{a}^{\prime} B \approx B / l_{\text {rad }}$, while the last source term in Eq. (18) is $\boldsymbol{\Omega} \cdot \nabla B \approx B / L$. In thin media, where $l_{\text {rad }} \gg L$, the first version of the source term is small, while in thick media, where $l_{\text {rad }} \ll L$, it is the other way around. In addition to the question of asymptotic behavior, the source terms in the difference formulation are smooth in the frequency domain as they do not involve a factor of $\sigma_{a}^{\prime}$.

The formulas in the previous section can be used to estimate the orders of the terms in Eq. (18) in optically thick regions. Let us divide the equation by $\sigma_{a}^{\prime} B$ and consider the $D / B$ term as the unknown. In thick media, the dominant source term is $|\boldsymbol{\Omega} \cdot \nabla B| / \sigma_{a}^{\prime} B \approx \epsilon_{\text {space }}$; therefore we conclude that $D / B \approx \epsilon_{\text {space }}$. We can then estimate that the other terms $(1 / c)(\partial B / \partial t) / \sigma_{a}^{\prime} B \approx \epsilon_{\text {time }} \approx \epsilon_{\text {space }}^{2}$ and $\boldsymbol{\Omega} \cdot \nabla D / \sigma_{a}^{\prime} B \approx \epsilon_{\text {space. }}^{2}$. Finally, the $(1 / c)(\partial D / \partial t) / \sigma_{a}^{\prime} B$ term is of order $\epsilon_{\text {space }}^{3}$.

Another significant difference between Eqs. (15) and (18) is in the angular dependence of the source terms. The source term in the traditional formulation is $\sigma_{a}^{\prime} B$; it is spherically symmetric, i.e. of $P_{0}$ symmetry. The dominant source term in the difference formulation is $\boldsymbol{\Omega} \cdot \nabla B$; it is antisymmetric in angle; more accurately it is of $P_{1}$ symmetry. In the difference formulation there is also a small source term, $(1 / c)(\partial B / \partial t)$ of $P_{0}$ symmetry. While the $\sigma_{a}^{\prime} B$ term adds energy to the radiation field, in the difference equation the dominant source term, $\boldsymbol{\Omega} \cdot \nabla B$, only transports the difference intensity; it adds nothing to the total energy of the radiation field. That task is relegated to the small $(1 / c)(\partial B / \partial t)$ term.

The source term in the traditional formulation for photon transport, $\sigma_{a}^{\prime} B$, accounts for spontaneous emission and is balanced by absorption in a thick system. In the difference formulation, the reference value for the radiation field is $B$, not zero. This reference value is a function of the local temperature, $T(\mathbf{x}, t)$, and is therefore a function of both space and time. The new source terms in the difference formulation have a straightforward, intuitive interpretation. The term involving the time derivative of $B$ can be understood from energy conservation. If the local temperature changes, the resultant change in $B$, all else remaining constant, must be accounted for by a change in the difference field, $D$, in order to maintain (locally) the energy in the radiation field.

The term involving the space derivative of $B$ is more interesting. To understand this term, consider transport in one-dimensional slab geometry where this term is now written $\mu d B / d x$; the direction cosine of the propagation di- 
rection is $\mu=\boldsymbol{\Omega} \cdot \hat{\mathbf{x}}$, where $\hat{\mathbf{x}}$ is a unit vector perpendicular to the slab. If the temperature is uniform, $d B / d x$ is zero and there are no sources. Consider, however, the case where there is a positive step in the value of $B$, of magnitude $b$, at the origin. The source term, $\mu d B / d x$, is now $\mu b \delta(x)$. The difference field has a source term only at the origin, with a negative source for positive $\mu$ and a positive source for negative $\mu$. The right-moving negative source is interpreted as the missing photons that would have been streaming across the origin if the step in $B$ did not exist. The negative sources are "photon holes", borrowing a term from solid state physics. The left-moving positive source is simply the photons being emitted from the hotter region into the cooler region. More succinctly, the $\mu d B / d x$ term generates the transport between the hotter and cooler regions that would otherwise not occur. The total "photon" energy emitted at the origin integrates to zero.

For completeness, we write the radiation energy density and its first moment, the radiation flux vector, in terms of $D$

$$
\begin{gathered}
E_{\text {rad }}=\frac{1}{c} \int_{0}^{\infty} d \nu \int_{4 \pi} d \boldsymbol{\Omega} I=\frac{1}{c} \int_{0}^{\infty} d \nu \int_{4 \pi} d \boldsymbol{\Omega}(D+B), \\
\mathbf{F}_{\text {rad }}=\int_{0}^{\infty} d \nu \int_{4 \pi} d \boldsymbol{\Omega} \boldsymbol{\Omega} I=\int_{0}^{\infty} d \nu \int_{4 \pi} d \boldsymbol{\Omega} \boldsymbol{\Omega} D,
\end{gathered}
$$

and the coupling of the radiation to the material, from Eq. (8)

$$
\frac{\partial E_{m a t}}{\partial t}=\int_{0}^{\infty} d \nu \int_{4 \pi} d \boldsymbol{\Omega} \sigma_{a}^{\prime} D+G .
$$

The energy conservation equation, (9), is unchanged.

\subsection{The diffusion limit, without scattering}

In thick media, in LTE, far from boundaries, after sufficient time, radiation tends to the diffusion limit. This is a well established result of asymptotic analysis; nevertheless even very recently a reanalysis was published by Morel [Mor00]. We show now how the difference formulation leads to the diffusion limit. In fact we will show it in two different ways. First, we formally integrate the transport equation; second, we show that the traditional asymptotic expansion yields the same result to first order. It has to be emphasized that we show the diffusion limit of the exact transport equation; therefore it includes all terms, it is able to satisfy boundary conditions correctly and it includes the treatment of boundary layers.

\section{Formal solution}

Equation (18) has a formal solution. We define a path variable, $s$, by

$$
\mathbf{x}=\mathbf{x}_{0}+\boldsymbol{\Omega} s \quad ; \quad t=t_{0}+s / c .
$$


It is easy to see that Eq. (18) can be written as

$$
\frac{d D}{d s}=-\sigma_{a}^{\prime} D-\frac{d B}{d s}
$$

giving the formal solution

$$
\begin{aligned}
D(s) & =D(0) \exp \left[-\int_{0}^{s} \sigma_{a}^{\prime}\left(s^{\prime}\right) d s^{\prime}\right] \\
& -\int_{0}^{s} d s^{\prime} \frac{d B\left(s^{\prime}\right)}{d s^{\prime}} \exp \left[-\int_{s^{\prime}}^{s} \sigma_{a}^{\prime}\left(s^{\prime \prime}\right) d s^{\prime \prime}\right] .
\end{aligned}
$$

The formal solution shows that the boundary condition, $D(0)$, decays in a few absorption lengths. Deep in the material $\sigma_{a}^{\prime}$ varies slowly. In fact both $\sigma_{a}^{\prime}$ and $d B / d s$ are constant to first order in $\epsilon_{\text {stream. }}$. Eq. (24) can then be integrated. The result is

$$
D(s)=\left[D(0)+\frac{1}{\sigma_{a}^{\prime}} \frac{d B}{d s}\right] \exp \left[-\sigma_{a}^{\prime} s\right]-\frac{1}{\sigma_{a}^{\prime}} \frac{d B}{d s} .
$$

It shows that

$$
D(s)=-\frac{1}{\sigma_{a}^{\prime}(s)} \frac{d B(s)}{d s}
$$

is the steady-state solution of Eq. (24) and that any boundary value of $D(0)$ decays to it in a few absorption lengths. A result that is correct to second order in $\epsilon_{\text {stream }}$ was given in [SB03].

\section{Asymptotic expansion}

The relative orders of various terms in the transport equation, Eq.(18), in thick media were estimated in Section 2.2. The estimation is valid far from boundary layers and time transients. To first order in $\epsilon_{\text {stream }}$, there are only two terms

$$
0=-\sigma_{a}^{\prime} D-\Omega \cdot \nabla B
$$

giving the solution

$$
D=-\frac{1}{\sigma_{a}^{\prime}} \boldsymbol{\Omega} \cdot \nabla B=-\frac{1}{\sigma_{a}^{\prime}} \frac{\partial B}{\partial T^{4}} \boldsymbol{\Omega} \cdot \nabla\left(T^{4}\right) .
$$

The radiation flux, from Eq. (20), is

$$
\mathbf{F}_{\text {rad }}=-\left[\int_{0}^{\infty} d \nu \frac{1}{\sigma_{a}^{\prime}} \frac{\partial B}{\partial T^{4}}\right] \frac{1}{3} \nabla\left(T^{4}\right)=-\frac{a c}{3 \sigma_{R}} \nabla\left(T^{4}\right) .
$$

This is the correct diffusion limit of the transport equation. We also recovered the correct definition of the Rosseland mean opacity, $\sigma_{R}$; see [Cas00], [MM84]. To first order Eq. (29) is identical to Eq. (25). It confirms the first 
order accuracy of the diffusion flux [Mor00]. Note the utter simplicity of the derivation.

An expansion in higher orders of $\epsilon_{\text {stream }}$ can also be carried out. The results are similar to those of Morel [Mor00], but they are slightly different and more consistent. A short discussion was given in [SB03].

\subsection{The difference formulation, in LTE, with scattering}

\section{The scattering term}

The scattering term was displayed in Eq. (5). In LTE, the Planck distribution at the material temperature is stationary and it also satisfies detailed balance. This imposes thermodynamic conditions on the scattering cross sections

$$
\begin{aligned}
& \frac{\nu}{\nu^{\prime}} \sigma_{s}\left(\nu^{\prime} \rightarrow \nu, \boldsymbol{\Omega} \cdot \boldsymbol{\Omega}^{\prime}\right) B\left(\nu^{\prime}\right)\left[1+\frac{c^{2} B(\nu)}{2 h \nu^{3}}\right]= \\
& \sigma_{s}\left(\nu \rightarrow \nu^{\prime}, \boldsymbol{\Omega} \cdot \boldsymbol{\Omega}^{\prime}\right) B(\nu)\left[1+\frac{c^{2} B\left(\nu^{\prime}\right)}{2 h \nu^{\prime 3}}\right],
\end{aligned}
$$

where the $\mathbf{x}, t ; T$ dependence of $\sigma_{s}$ and $B$ has been suppressed. After some algebra we get the surprising result

$$
\begin{aligned}
Q(D) & =\int_{0}^{\infty} d \nu^{\prime} \int_{4 \pi} d \boldsymbol{\Omega}^{\prime} \frac{\nu}{\nu^{\prime}} \sigma_{s}\left(\nu^{\prime} \rightarrow \nu, \boldsymbol{\Omega} \cdot \boldsymbol{\Omega}^{\prime}\right) D\left(\nu^{\prime}, \boldsymbol{\Omega}^{\prime}\right)\left[1+\frac{c^{2} D(\nu, \boldsymbol{\Omega})}{2 h \nu^{3}}\right] \\
& -\int_{0}^{\infty} d \nu^{\prime} \int_{4 \pi} d \boldsymbol{\Omega}^{\prime} \sigma_{s}\left(\nu \rightarrow \nu^{\prime}, \boldsymbol{\Omega} \cdot \boldsymbol{\Omega}^{\prime}\right) D(\nu, \boldsymbol{\Omega})\left[1+\frac{c^{2} D\left(\nu^{\prime}, \boldsymbol{\Omega}^{\prime}\right)}{2 h \nu^{\prime 3}}\right] .
\end{aligned}
$$

We would like to stress that Eq. (31) is valid only in LTE. Otherwise the stimulated scattering terms cannot be written in terms of $D$ alone.

If scattering does not change the radiation energy, e.g. in Thomson scattering, the stimulated emission terms in Eq. (5) cancel identically and an isotropic distribution is stationary under those conditions. In fact, we define, as usual

$$
J(\mathbf{x}, t ; \nu):=\frac{1}{4 \pi} \int_{4 \pi} d \boldsymbol{\Omega} I(\mathbf{x}, t ; \nu, \boldsymbol{\Omega}) .
$$

Then $I=J$ is stationary and Eq. (5) can be written as

$$
\begin{aligned}
Q_{\text {mono }}(I-J) & =\int_{4 \pi} d \boldsymbol{\Omega}^{\prime} \sigma_{s}\left(\nu, \boldsymbol{\Omega} \cdot \boldsymbol{\Omega}^{\prime}\right)\left[I\left(\nu, \boldsymbol{\Omega}^{\prime}\right)-J(\nu)\right] \\
& -\int_{4 \pi} d \boldsymbol{\Omega}^{\prime} \sigma_{s}\left(\nu, \boldsymbol{\Omega} \cdot \boldsymbol{\Omega}^{\prime}\right)[I(\nu, \boldsymbol{\Omega})-J(\nu)] .
\end{aligned}
$$

In the scattering terms, Eqs. (5), (31), (33), both $\sigma_{s}\left(\nu^{\prime} \rightarrow \nu, \boldsymbol{\Omega} \cdot \boldsymbol{\Omega}^{\prime}\right)$ and $I(\nu, \boldsymbol{\Omega})$ or $D(\nu, \boldsymbol{\Omega})$ can be expanded in spherical harmonics [Brn95]. The integrals are then reduced to relaxation equations for the spherical harmonic 
components of $D$, or $I-J$, respectively. In particular, if the scattering is isotropic, Eq. (33) reduces to

$$
Q_{\text {mono }}(I-J)=\sigma_{s}(\nu)(I-J) .
$$

Finally, we note that the scattering terms are always proportional to

$$
\sigma_{s}(\nu)=\int_{4 \pi} d \boldsymbol{\Omega}^{\prime} \sigma_{s}\left(\nu, \boldsymbol{\Omega} \cdot \boldsymbol{\Omega}^{\prime}\right),
$$

and to the analogous expressions in Eqs. (5), (31).

\section{The full equations}

The full equations in the difference formulation, in LTE, are obtained by adding the right-hand side of Eq. (31) to the right-hand side of Eq. (17). As the change of the radiation energy caused by scattering comes from the matter, the integral of Eq. (31) over frequency has to be subtracted from Eq. (21), in analogy to Eq. (8). They have to be solved together with the conservation equations, Eq. (9). In the more general case they have to be solved together with the full set of equations of radiation hydrodynamics [Cas00], [MM84], [Pom73].

Rather than presenting the formal development of the equations, we will sketch their simplified version that gives some insight into the relaxation behavior of the radiation intensity. We start by rewriting Eq. (1) in terms of the path variable, $s$, as defined in Eq. (22). We also take liberties with the scattering term; we tacitly assume it to be monochromatic and isotropic, as in Eq. (34).

$$
\frac{d I}{d s}=-\sigma_{a}^{\prime}(I-B)-\sigma_{s}(I-J) .
$$

We define the total extinction coefficient, $\sigma_{t}=\sigma_{a}^{\prime}+\sigma_{s}$. Obviously,

$$
\left[\frac{\sigma_{a}^{\prime}}{\sigma_{t}}+\frac{\sigma_{s}}{\sigma_{t}}\right] \frac{d I}{d s}=-\sigma_{a}^{\prime}(I-B)-\sigma_{s}(I-J) .
$$

In analogy to the difference formulation, we subtract appropriate terms from the equation to give

$$
\begin{array}{r}
\frac{\sigma_{a}^{\prime}}{\sigma_{t}} \frac{d(I-B)}{d s}+\frac{\sigma_{s}}{\sigma_{t}} \frac{d(I-J)}{d s}= \\
-\sigma_{a}^{\prime}(I-B)-\sigma_{s}(I-J)-\frac{\sigma_{a}^{\prime}}{\sigma_{t}} \frac{d B}{d s}-\frac{\sigma_{s}}{\sigma_{t}} \frac{d J}{d s} .
\end{array}
$$

Depending on the relative magnitudes of $\sigma_{a}^{\prime}$ and $\sigma_{s}$, it is easy to see that asymptotically, in thick media, the equation approaches either the diffusion approximation or the Eddington approximation. 
An alternative rearrangement gives

$$
\frac{d I}{d s}=-\sigma_{a}^{\prime}(J-B)-\left(\sigma_{a}^{\prime}+\sigma_{s}\right)(I-J)
$$

We now subtract $d B / d s$ from both sides of the equation, to give

$$
\frac{d(I-J)}{d s}+\frac{d(J-B)}{d s}=-\sigma_{a}^{\prime}(J-B)-\left(\sigma_{a}^{\prime}+\sigma_{s}\right)(I-J)-\frac{d B}{d s} .
$$

With the further assumption of $d B / d s=0$ and the constancy of $\sigma_{a}^{\prime}$ and $\sigma_{s}$ along the radiation path, it is easy to verify that Eq. (40) has the solution

$$
\begin{gathered}
J-B=[J(0)-B(0)] \exp \left[-\sigma_{a}^{\prime} s\right], \\
I-J=[I(0)-J(0)] \exp \left[-\left(\sigma_{a}^{\prime}+\sigma_{s}\right) s\right] .
\end{gathered}
$$

We note at this point that the material relaxation equation, Eq. (8), can always be written as

$$
\frac{\partial E_{m a t}}{\partial t}=4 \pi \int_{0}^{\infty} d \nu \sigma_{a}^{\prime}(J-B)+G,
$$

so $J-B$ is a natural variable to consider.

We emphasize again that our derivation of the solution of Eq. (40) was highly simplistic. In particular it did not take into account material relaxation and the possibility of scattering with change of photon frequency, e.g. Compton scattering [Cas00]. We hope to report later on further developments along these lines.

\section{Test Problems}

A variety of test problems have been investigated in [Bro05] in order to evaluate the computational efficiency and accuracy of the difference formulation, employing the Symbolic Implicit Monte Carlo (SIMC) method. Our goal was to analyze some simple situations that indicate the potential impact of the difference formulation in more complex physics environments. The key issues are accuracy and efficiency for both thick and thin media, and specfically the frequent occurence of media which are thick at one frequency while being thin at others. In addition to comparing the Monte Carlo solution of the two formulations of transport, we compared them to analytic, or semi-analytic solutions where they are available. The main results of that study will be summarized below. 


\subsection{Behavior of a finite slab heated from the outside}

A very basic test of a time-dependent thermal transport algorithm is whether it correctly approaches the steady state solution for a finite slab immersed in a heat bath with different temperatures on either side. The fourth power of the temperature should become a linear function of the optical depth in a gray, thick medium, in steady state. Deviations from such a straight line are indicative of boundary layers when they occur within a mean free path or so of a surface, but otherwise indicate serious problems in the numerical solution. Teleportation errors result in a wrong slope and cause curvature in the interior solution [MBS03]; for this reason zone thickness were limited to one mean free path in our piecewise constant treatment of the material temperature.

In addition to the issues noted above, the time dependent approach to steady state offers the opportunity to check the correctness of the implementation of interior source terms as well as initial and boundary conditions. That the steady state temperature is independent of time step, reflecting implicit behavior of the time integration, can also be checked. When the spectrum of the radiation field is examined, even for a grey opacity, the correctness of the frequency sampling algorithm can be checked. Agreement between the standard and difference formulation is non-trivial due to the different nature of the source terms.

In the presentation of our computational results below, we provide a clear demonstration of rigorous agreement between the two formulations for transport, in terms of their approach to steady state, along with a measure of increase in computational efficiency for the difference formulation. The magnitude of this increase in computational efficiency, in the form of greatly reduced Monte Carlo noise as the optical thickness of the problem is increased, is somewhat surprising even to the authors who were prospecting for it.

In the first set of simulations we considered a finite slab heated from the left side, with an open boundary on the right, allowing the radiation flowing through the slab to enter free space. The slab was composed of a uniform, static material having a frequency-independent (gray) opacity. We calculated the time dependence of the temperature and of the radiation field after a 1 $\mathrm{keV}$ black-body source on the left side of the slab is turned on at time $t=0$. $\left(1 \mathrm{keV} \approx 1.210^{7}{ }^{\circ} \mathrm{K}\right.$.)

During the time dependent execution of the problem, a thermal wave, also known as Marshak wave, sweeps the problem domain and the solution then approaches steady state. We compared the solutions provided by the two formulations and their relative noise, for identical problem run times, in order to obtain a measure of the accuracy and relative computational efficiency of the two formulations for transport, under conditions that the Monte Carlo portion of the code dominates execution time.

Four instances of this problem are presented below. The slab is composed of a uniform material having a frequency independent (gray) opacity of $0.1,1$, 10 , and 100 mean free paths per $\mathrm{cm}$ respectively. The slab is $10 \mathrm{~cm}$ thick so the 
four opacities correspond to total optical depths of 1, 10, 100 and 1000 mean free paths. The specific heat of the material is a constant 0.1 jerk $/\left(\mathrm{keV} \mathrm{cm}^{3}\right)$, where jerk is an energy unit ( 1 jerk $=10^{16}$ ergs), and temperature is measured in energy units of $\mathrm{kT}=1 \mathrm{keV}$. The slab is initially at a temperature of $0.01 \mathrm{keV}$, with the radiation field being a Planckian in equilibrium with this temperature. All four problems used a time step of $0.2 \mathrm{sh}$, where $1 \mathrm{sh}=$ $10^{-8}$ sec. The problem 1 mean free path thick was run to $20 \mathrm{sh}$ in order to get close to steady state, as was the problem 10 mean free paths thick. The problem 100 mean free paths thick was run to 40 sh in order to approach steady state. The problem 1000 mean free paths heated up very slowly due to the diffusive nature of the solution, requiring 320 sh in order to suitably approach steady state. The problems 1 and 10 mean free paths thick employed 20 zones, while the thicker problems employed zones one mean free path thick in order to prevent teleportation error from influencing the results, and to prevent anomalous performance results for the difference formulation. Equal thickness zones were used everywhere. Geometric zoning has a role only if piecewise linear treatment of the material temperature is available to remove teleportation error.

The speed of light is $300 \mathrm{~cm} / \mathrm{sh}$. This provides 6 traversals of the slab for a time step of $0.2 \mathrm{sh}$, reflecting on the stability of the method. Relaxing the need for implicit treatment of the source terms was one of the hopes of the authors, given that implicit treatment requires the solution of a non-linear system of equations for each time step. Our experiences in this regard, documented in [Daf05], were made even more difficult by the $T^{4}$ term for thermal emission. Explicit treatment of the $\partial B / \partial x$ source terms was abandoned as a result, but may be worth revisiting in mixed physics applications where the time step size is limited for reasons outside of transport physics.

We would like to note that the Monte Carlo solution for the two transport formulations have entirely different requirements for spatial importance sampling if uniform statistical noise, as a function of position, is to be obtained. In the standard formulation most of the computational effort is spent computing the balance between emission and absorption that produces the local equilibrium black body field. As a result, a scheme that samples particles with a uniform density in space produces a relatively flat statistical noise across the slab. The number of source particles born in each zone, during each time step, is proportional to the thickness of the zone. The statistical properties of the Monte Carlo solution for the difference formulation are in sharp contrast to this. For thicker problems, the statistical noise for the difference formulation is miniscule near the hot side and increases very rapidly towards the cold boundary. Suitable importance sampling could flatten out this growth in noise. An exposition of this topic is beyond the scope of this paper.

In the results shown below, the unit of source sampling was one $\partial B / \partial t$ particle, and one $\partial B / \partial x$ particle pair, for each zone, or zone interface, respectively, for the difference formulation. We have not attempted to tune the ratio between $\partial B / \partial t$ and $\partial B / \partial x$ source particles, nor have we attempted to tune 
the relative importance of source sampling across the volume of the problem. In the standard formulation, the unit of source sampling is one thermally emitted particle per zone.

In Figure 1 (a) we show the steady state solution for the material temperature, as a function of position in the slab, for the problem instance that is one mean free path thick. The average of 100 randomly seeded runs using the standard formulation, and the average of the same number of instances using the difference formulation, is shown by the boxes and the $\times$ symbols, respectively. The two formulations are in perfect agreement. The standard deviation of the results for the standard formulation, as well as the same statistic for the difference formuation, both multiplied by 400 , are plotted using diamonds and triangles, respectively. The Monte Carlo source particle counts were selected for equal run times for the two formulations, and were high enough that the cost of Monte Carlo transport dominated execution time. The relative performance of the methods, then, is the ratio of the squares of the standard deviations. There is a small advantage in favor of the difference formulation, except at the very right hand side of the slab. Note that the optical thickness of each zone is only 0.05 optical depths.

In Figure 1 (b) we show the results for a slab that is 10 mean free paths thick. Again, we obtain perfect agreement for the two formulations when the average of the equilibrium material temperature is examined. In order to see the standard deviation for the two formulations on the same plot, we now have to apply a scale factor of 100 for the standard formulation and 1000 for the difference formulation. The trend of growth for the standard deviation for the difference formulation, as the temperature gradient increases in the slab from left to right, is becoming apparent.

In Figure 1 (c) we show the results for a slab that is 100 mean free paths thick. There are 100 zones in this problem in order to avoid teleportation error, with every fifth point plotted using a symbol. The other points are included in the solid lines drawn. The growth in noise for the standard formulation is now becoming visible in the plot. We apply a scale factor of only 10 to see the standard deviation for the standard formulation. The growing trend of the standard deviation for the difference formulation, as one traverses the slab from left to right is now quite clear, but the computational advantage for the difference formulation is large.

In Figure 1 (d) we show the results for a slab that is 1000 mean free paths thick. Thee are 1000 zones in this problem, a requirement to avoid significant teleportation error, with every 50 'th zone plotted using a symbol. The disagrement between the two formulations appearing towards the left side of the slab are statistical fluctuations. The computational advantage of the difference formulation is extreme.

At this point, the reader may note that the results for the difference formulation have the appearance of smooth curves, regardless of the optical thickness of the problem. The $1 / \sqrt{N}$ noise behavior typical of a Monte Carlo 
solution is still present, it is just that the amplitude of the noise is small and remains small as the optical thickness of the problem increases.

Evidence of the scaling behavior of the computational advantage is shown in the next figure. As noted above, the number of Monte Carlo particles for each optical thickness is set so that the difference formulation and standard formulation exhibit the same run time, and that the computer time is dominated by the Monte Carlo, so that the standard deviation is scaling like one over the square root of the computational work. Under these conditions, the relative computational efficiency of the two methods is the ratio of the squares of their standard deviations. In Figure 2 we show the computational advantage exhibited in the standard deviation of the material temperature, as a function of position, for the four optical thicknesses. The computatational advantage scales, roughly, as the square of the optical depth of the problem.

Finally, in Figure 3 we show the penetration of the thermal wave into the material, 1000 optical depths thick, at an intermediate time. As in Figure 1 (d), the material temperature is plotted vs. distance. Refining the time step and zone size demonstrates that the solution shown is fully converged. This figure illustrates the gains that the difference formulation provides, clearly visibly to the reader.

\subsection{Comparison to analytic diffusion solution for a linearized problem}

In order to check the correctness of our numerical implementation, we have compared results with the diffusion solution appearing in [SO96]. This solution requires that the material energy take the form

$$
E_{m a t}=\frac{\alpha T^{4}}{4}
$$

or, equivalently, a specific heat of the form

$$
\frac{\partial E_{m a t}}{\partial T}=\alpha T^{3} .
$$

The purpose of this form for the material energy is to remove the non-linearity, $T^{4}$, that otherwise prevents an analytic solution. The resulting analytic solution can then be used to check for correct convergent behavior in the numerical simulation. The behavior of the specific heat at $T=0$, however, makes things quite fragile unless the numerical code itself is transformed to handle things in linearized form. To some extent, this defeats the purpose of checking the original code, much of which had to be modified to resolve the problem.

For this problem, $\alpha$ and the cross section were chosen so that the values in the tables of analytical results appearing in [SO96] could be used directly. In Figure 4 we plot the material temperature produced by our Monte Carlo solution of the standard and difference formulation, along with data from $\mathrm{Su}$ and 
Olson's analytical solution corresponding to a late time, $\tau=10$. We obtain good agreement where analytical data are available. This is expected because for $\tau=10$ the diffusion approximation assumed by Su and Olson is valid. In Figure 5 we plot the material temperature for the same problem at a much earlier time, along with data from $\mathrm{Su}$ and Olson's analytical solution for a $\tau=.01$. In this case, not surprisingly, there is sharp disagreement between the diffusion solution and the fully overlapped and converged Monte Carlo transport solutions for the standard and the difference formulation. Here the fundamental limitation is the speed of light. It is fully respected by the transport solution but ignored by the diffusion solution.

\subsection{Time dependent Marshak wave problem, with a non-trival opacity}

Our Monte Carlo solutions of the standard and difference formulations of transport fully implement the details of the thermal frequency spectrum; the spectral properties of the derivative sources make the agreement between the two formulations non-trivial even in the case of a gray opacity. Once the spectral sampling of the source terms in the difference formulation is done correctly, as described in Appendix A of [Bro05], there is no more to be done for the correct treatment of a frequency dependent opacity in the difference formulation other than to use the correct absorption cross section for the given Monte Carlo particle. The accuracy of the treatment of the frequency dependent cross section is as good as the cross section itself.

The emission term for the standard formulation, on the other hand, appears as $\sigma B$ and in the most general case it must be numerically integrated across a frequency group structure in each zone, for the temperature at the start of each time step, in order to produce the probability distribution for the emission spectrum that, then, must be sampled. This requirement provides another place where numerical errors must be controlled in the implementation of the standard formulation.

We now turn to a relatively simple frequency dependent opacity. The opacity is constant (gray and 1000 mean free paths for the slab) for frequencies below $1 \mathrm{keV}$ and again constant (gray and 10 mean free paths for the slab) for frequencies above $1 \mathrm{keV}$. This corresponds, roughly, to the precipitous drop in opacity that can occur in real materials, as a function of frequency. The portion of the emitted spectrum below $1 \mathrm{keV}$ is strongly re-absorbed, while the portion of the emitted spectrum above $1 \mathrm{keV}$ encounters a lower opacity and transports freely. This is a difficult non-linear problem because, as the trapped radiation heats the material, the Planckian emission spectrum moves towards higher frequencies where radiation flows freely.

In Figure 6 we show the material temperature for one instance of this problem, at an intermediate time where the thermal wave is still propagating through the slab. Again, as before, the standard formulation is the one 
exhibiting a high level of statistical noise. Note, however, that this noise disappears in the "foot" of the advancing thermal wave. The reduction in noise for the standard formulation is due to the fact that this feature is caused by photons at high frequencies where the material has a reduced opacity. Those photons are absorbed, but not reemitted by the cold matter.

\section{Summary and directions for further work}

\subsection{Theory}

In earlier work [SB03], a new analytical formulation was introduced for the transport equation. The new formulation is for the transport of the difference between the specific intensity and the local black body equilibrium radiation at the matter temperature, at any point in space, time and direction. Appropriately, we called the new transport equation the difference formulation to distinguish it from the traditional formalism. We have shown that the difference formulation is expressed in terms of quantities that become small in optically thick media. The transformation is a simple one and results in a completely equivalent system of equations, without approximation.

The most important distinction between the two formulations is in the source terms. In the traditional formulation, the source term is the spontaneous emission of the medium. It is small in optically thin regions, resulting in straight line propagation of photons. The traditional formulation is well suited for this regime. In the difference formulation, the source term is the space-time gradient of the Planck function at the material temperature. The latter gets small in optically thick regions. In addition to this important difference in asymptotic behavior, the two formulations differ in that the spontaneous emission depends upon the absorption cross sections for the emitting medium, while the source term in the difference formulation depends only upon the temperature of the medium, as a function of space and time. The two formulations are able to satisfy equivalent boundary conditions and initial conditions.

Even the largest terms in the difference formulation are of the order of $\epsilon_{\text {space }}$, i.e. the ratio of the photon mean free path to the gradient length. In optically thick regions this ratio is a small quantity. We have shown that the equations reduce to the diffusion limit in the proper circumstances. We have also discussed briefly the extensions needed when scattering is important.

\subsection{Computations}

The Symbolic Implicit Monte Carlo method, SIMC, [Bro86] is an attractive framework for the calculation of radiation transport in complex media and geometries; it provides a basis for accurate and stable numerical schemes [Nka91] 
[DL04]. In our previous paper, [Bro05], we have demonstrated that the difference formulation [SB03] is eminently suitable for numerical simulations of radiation transport, employing the SIMC technique.

Theoretical expectations were that the traditional formulation would be good for thin regions, while the difference formulation would be advantageous in thick media. We have demonstrated that the difference formulation, when employing the SIMC technique, offers significant noise reduction for thick systems. The expected cross-over vis-a-vis the standard formulation occurs for very thin systems, thin enough that the difference formulation might become a panacea for Monte Carlo treatment of thermal radiation transport. Its advantage for thick systems goes like the square of the optical depth of the system.

The character of the source terms is very different in the traditional and the difference formulations: thermal emission in the former is replaced by derivative source terms in the latter. Therefore a key issue for the accuracy and stability of the difference formulation is the successful treatment of those derivative source terms. We have developed efficient, accurate analytic techniques for sampling the frequency spectrum of the source terms for the difference formulation. Frequency sampling in the difference formulation depends only on the space and time derivative of the material temperature, not on detailed material optical properties. This offers a significant advantage for problems with complicated material optical properties. The gain in terms of code complexity became clear when we implemented the test problem with the step in opacity. Although the test problems presented were very simple, in order to be able to clearly identify the advantages of the method, extensions to more complex situations do not present conceptual difficulties.

We would like to note that the computational gain for the difference formulation demonstrated in this paper is for the Monte Carlo portion of the problem. In order to obtain implicit treatment of the source terms, the SIMC technique requires the solution of a non-linear system of equations in order to perform the temperature update at the end of the time step. The cost of this can become significant when the number of zones in the problem is large. We used band-limited Gaussian elimination in our numerical work on problems with as many as 1000 zones. The bandwidth was limited both by the time step size and by the death of Monte Carlo particles when they became too small, relative to their birth weight. Multi-dimensional problems with significantly larger numbers of zones will pose a challenge, requiring the use of suitable iterative solution techniques. We believe that the demonstrated noise reduction in thick systems will be worth the effort involved.

We would like to note that our test problems have been relative simple, suitable as the first numerical tests for the difference formulation for thermal radiation transport. In our presented results, it is clear that the importance sampling requirements for the difference formulation are quite different than those for the standard one. It is also the case that improvements such as weight vectors in frequency space, and deterministic handling of the spectral output 
from the interface adjoining free space, have a significant impact on noise when spectral information associated with the photon field is desired. The details of these enhancements, and their relative value, are dependent upon the exact nature of the problems being run and the computational results that are desired.

\subsection{Work in progress}

We believe that the difference formulation will help in numerical solutions of the equations of radiation hydrodynamics in optically thick regions. We expect that it will be useful regardless of the numerical method employed, be it a deterministic method, for example $S_{n}$ and $P_{n}$, or a Monte Carlo method, for example the Symbolic Implicit Monte Carlo (SIMC) method of Brooks [Bro86], employed here. The dominant source of instability for Monte Carlo methods, the spontaneous emission term, is removed in the difference formulation and replaced by terms that are small in thick systems. Because of this, the well known stability problem for Monte Carlo methods in thick systems may, in fact, be removed. We will report on this possibility in future work. We would like to note that the Symbolic Implicit Monte Carlo method is well suited for dealing with the $(1 / c)(\partial B / \partial t)$ term, should it be a source of instability. Preliminary results show that the efficiency for Monte Carlo methods in thick systems will be improved, due to the removal of the balance between emission and absorption in a zone that produces a relatively noisy estimate for the difference.

A similar treatment may be useful in other transport problems. Neutron transport near criticality has many of the same properties as photon transport in optically thick regions. Similarly, the success of radiation therapy depends on accurate modeling of particle transport in the presence of strong absorption and scattering. We hope to be able to extend our treatment to some of those applications in the future.

In our numerical work on the difference formulation, we have employed a piecewise constant discretization for the material temperature. Due to teleportation effects, this discretization does not provide the correct diffusion limit for zone sizes that are large compared to the mean free path of a photon. It was clearly established by Clouet and Samba [CS04] that a piecewise linear treatment of the material temperature is required to obtain the correct diffusion limit, thereby eliminating the teleportation problem [MBS03]. In view of our results so far, we expect that introducing such treatment will enable the SIMC method to treat opically thick media with the same efficiency as optically thin ones. In addition, as discussed in the paper, the two formulations are completely equivalent. That suggests that they can be freely mixed within a single problem. This should enable one to take the best advantage of either formulation. We expect that the result will be an accurate and efficient treatment of radiation transport in almost any medium. 
Further studies in progress include a study of the numerical stability of various methods. We also intend to generalize the difference formulation to include non-monochromatic scattering and investigate its applicability to some well known approximate treatments, e.g. the Eddington approximation and the Kompaneets equation.

\section{References}

[Mih78] Mihalas, D.: Stellar Atmospheres, Freeman, San Francisco, pp 64-71 (1978)

[FC71] Fleck, J.A. and Cummings, J.D.: An implicit Monte Carlo scheme for calculating time and frequency dependent radiation transport, J. Comput. Phys. 8, 313 (1971).

[PB83] Larsen, E. W., Pomraning, G. C. and Badham, V. C.: Asymptotic analysis of radiative-transfer problems, J. Quant. Spectr. Rad. Transfer 29, 285 (1983).

[Pom82] Pomeraning, G.C.: Flux Limiters and Eddington Factors, J. Quant. Spectr. Rad. Transfer 27, 517-530.

[Mrs47] Marshak R.E.: Note on the Spherical Harmonic Method as Applied to the Milne Problem for a Sphere, Phys. Rev. 71 443-446 (1947)

[Mrk47] Mark, J.C.: The spherical harmonic method, Technical Report, CRT-340, Atomic Energy of Canada, Ltd., Ontario, 1947.

[Bro86] Brooks, E.D.: Symbolic Implicit Monte Carlo, J. Comput. Phys. 83, 433446 (1989).

[Nka91] N'kaoua, T.: Solution of the nonlinear radiative transfer equations by a fully implicit matrix Monte Carlo method coupled with the Rosseland diffusion equation via domain decomposition, SIAM J. Sci. Stat. Comput. 12, 505 (1991).

[DL04] Densmore J.D., Larsen E. W.: Asymptotic Equilibrium Diffusion Analysis of Time-Dependent Monte Carlo Methods for Grey Radiative Transfer, J. Comput. Phys. 199, 175-204 (2004)

[CF73] Carter, L.L. and Forest, C.A.: Nonlinear radiation transport simulation with an implicit Monte Carlo method, LA-5038, Low Alamos National Laboratory, 1973 (unpublished).

[SB03] Szőke, A. and Brooks, E.D.: The transport equation in optically thick media, J. Quant. Spectr. Rad. Transfer 91, 95-110 (2005)

[Bro05] Brooks, E.D, et al.: Symbolic implicit Monte Carlo radiation transport in the different formulation: a piecewise constant discretization, J. Comput. Phys. (in press, available online) (2005)

[Cas00] Castor, J.I.: Lectures on Radiation Hydrodynamics, UCRL-JC-134209, 2000 .

[MM84] Mihalas, D. and Mihalas, B. W.: Foundations of Radiation Hydrodynamics, (New York: Oxford University Press), 1984.

[Pom73] Pomraning, G. C.: The Equations of Radiation Hydrodynamics, (Oxford: Pergamon), 1973.

[MA00] Mihalas, D. and Auer, L.H.: On laboratory-frame radiation hydrodynamics, J. Quant. Spectr. Rad. Transfer, 7 61-97 (2000). 
[Mor00] Morel, J.E.: Diffusion-limit asymptotics of the transport equation, the $P_{1 / 3}$ equations, and two flux-limited diffusion theories, J. Quant. Spectr. Rad. Transfer 65 769-778 (2000).

[Brn95] Brown, P. N.: A linear algebraic development of diffusion synthetic acceleration for 3-dimensional transport-equations, SIAM Journal of Numerical Analysis, 32 179-214 (1995).

[MBS03] McKinley, M. S., Brooks, E. D, and Szoke, A.: Comparison of Implicit and Symbolic Implicit Monte Carlo Line Transport with Frequency Weight Vector Extension, J. Comput. Phys. 205, 330-349 (2003)

[Daf05] Daffin, F., et al.: An Evaluation of the Difference Formulation for the Transport of Atomic Lines, J. Comput. Phys. 204 27-45 (2005)

[SO96] Su, B. and Olson, G.L.: Benchmark results for the non-equilibrium marshak diffusion problem, J. Quant. Spectr. Rad. Transfer 56, 337-351 (1996).

[CS04] Clouet, J.-F. and Samba, G.: Asymptotic diffusion limit of the symbolic Monte-Carlo method for the transport equation J. Comput. Phys. 195 293-319 (2004) 

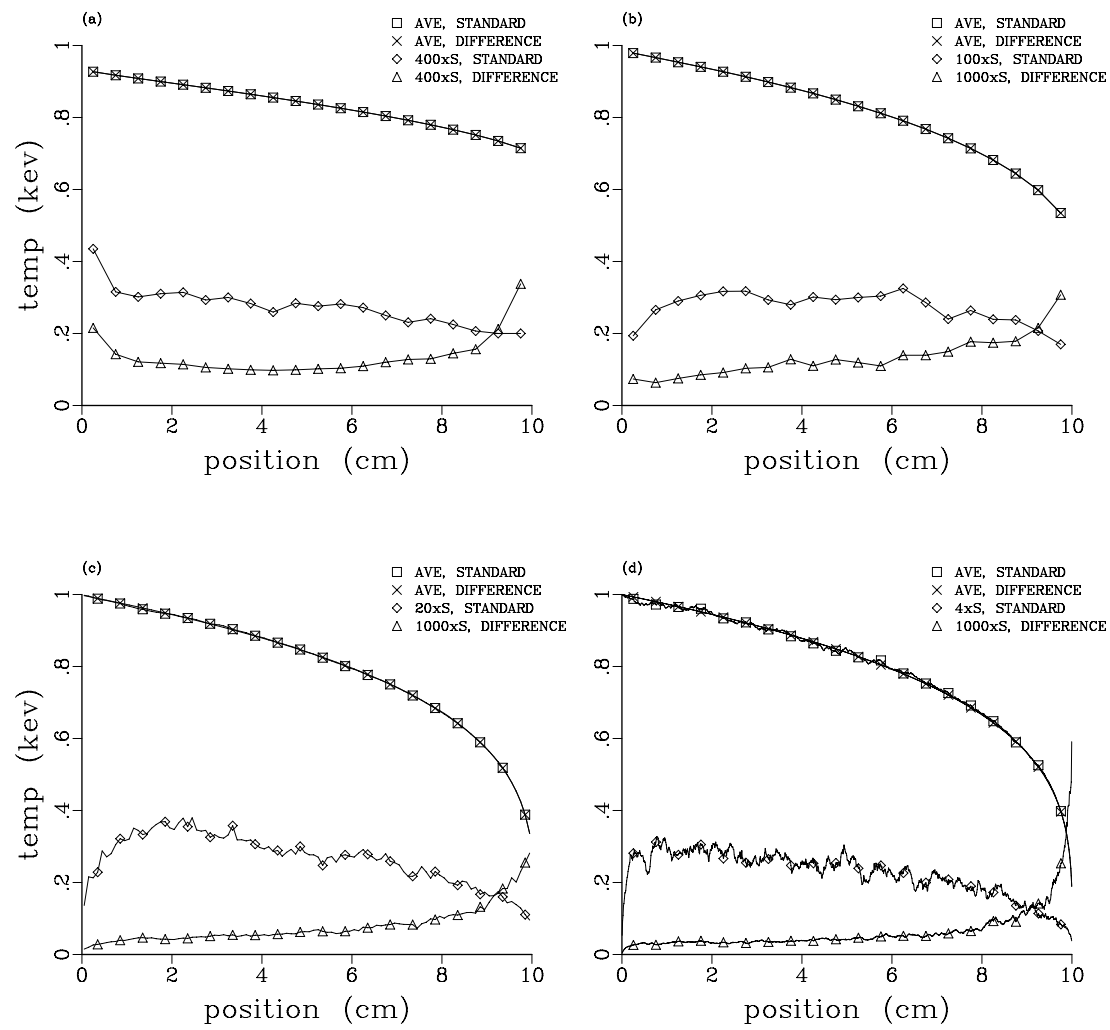

Fig. 1. Temperature distribution in a slab in steady state. The slab is heated from the left by a $1 \mathrm{keV}$ black body, and it radiates freely on the right. The total optical depth (OD) of the slab is 1 in (a), 10 in (b), 100 in (c) and 1000 in (d). The standard deviation of the standard formulation is denoted by diamonds and that of the difference formulation by triangles. Note the change in their relative scale with optical depth. The noise in the standard formulation increases dramatically with optical depth, while in the difference formulation it does not. 


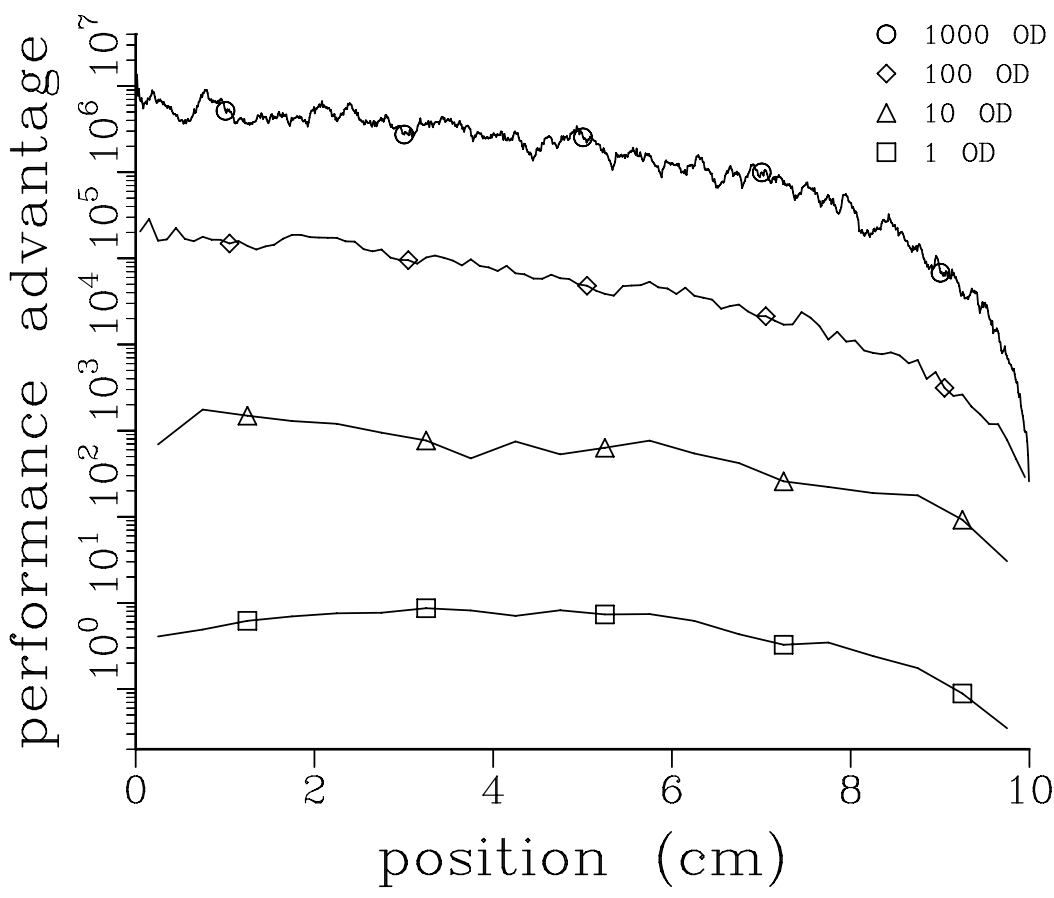

Fig. 2. The relative computational advantage of the difference formulation compared to that of the standard formulation, plotted as a function of position for various optical depths of the slab. In the 1 OD case, each zone is only $1 / 20 \mathrm{OD}$ thick, nevertheless, the difference formulation is better than the standard one except near the surface of the slab on the right hand side. There is a sharp decrease in computational advantage where the temperature gradient is large. This could easily have been remedied by spatial importance sampling of the source particles. 


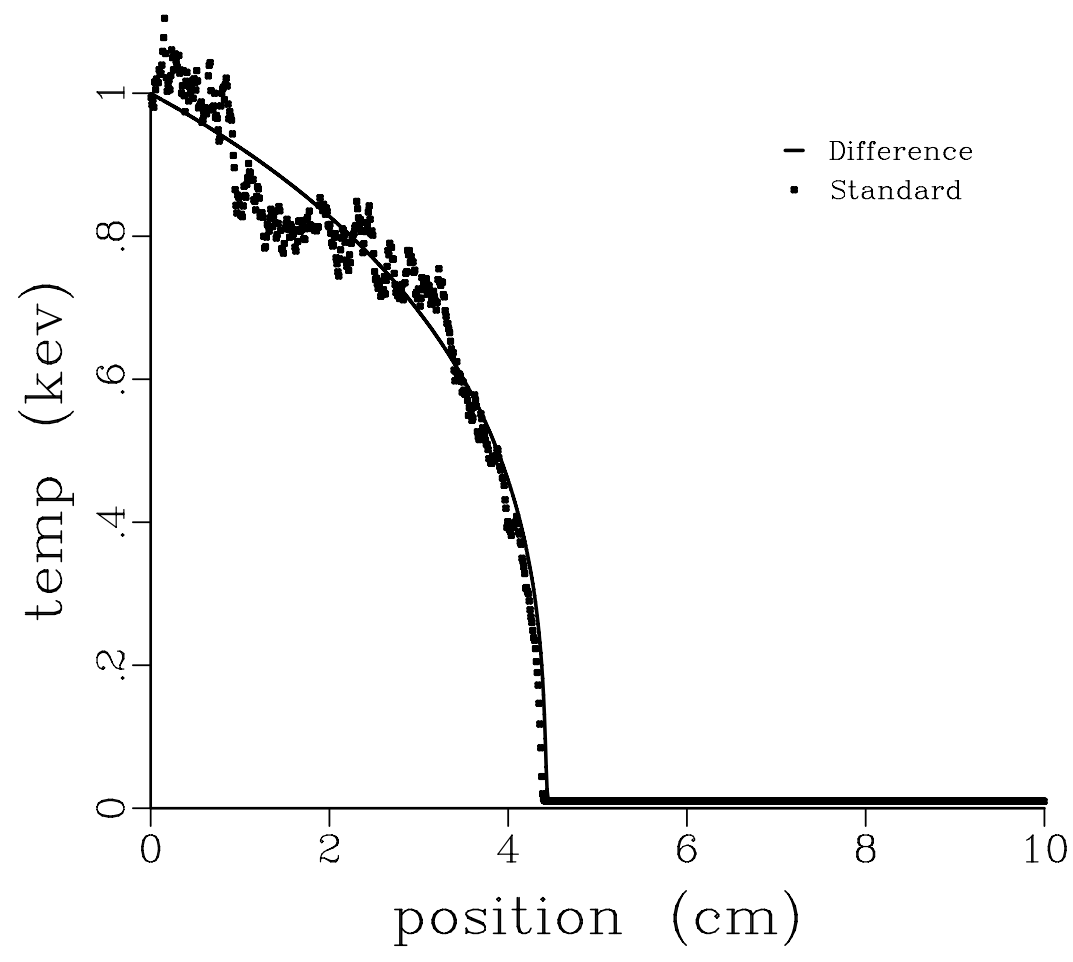

Fig. 3. Thermal wave (Marshak wave) penetrating a uniform, gray slab of 1000 OD at an early time, $40 \mathrm{sh}$. The standard formulation gives a noisy temperature profile whereas that of the difference formulation is many orders of magnitude smoother. The slight difference in the position of the leading edge is a statistical fluctuation for the standard formulation. 


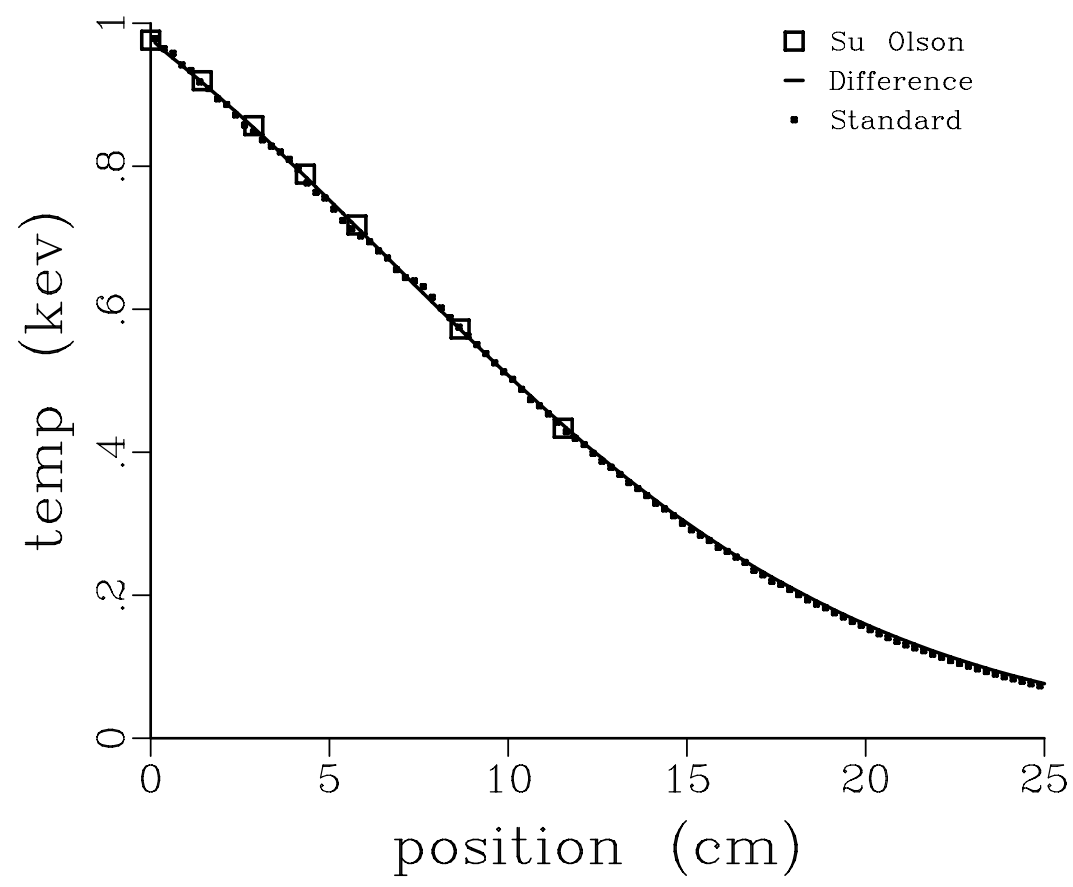

Fig. 4. Temperature distribution in the $\mathrm{Su} \&$ Olson problem [SO96] at a late time $\tau=10$. There is excellent agreement of the calculations with analytic results. 


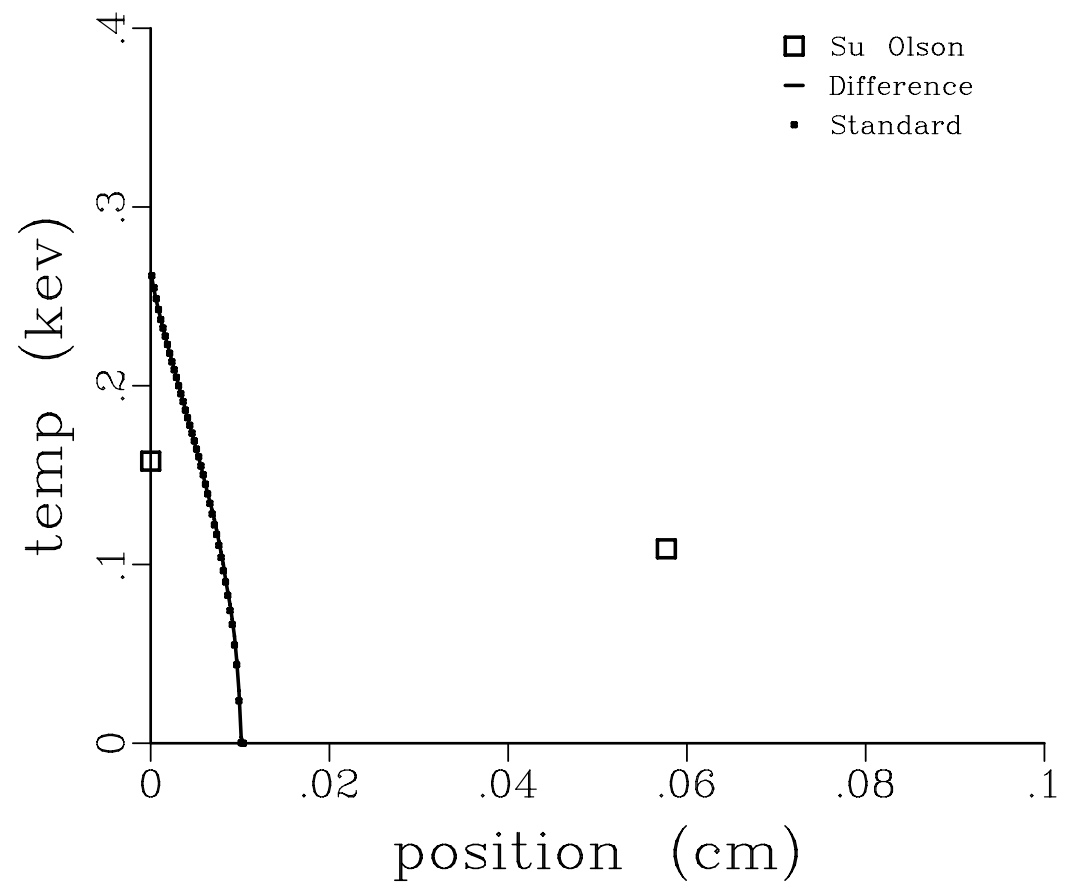

Fig. 5. Temperature distribution in the $\mathrm{Su} \&$ Olson problem [SO96] at an early time $\tau=0.01$. The transport solution is limited by the speed of light, that is 1 in the units of $\tau$. Note that there is no significant spreading of the radiation front at the leading edge of the thermal wave. 


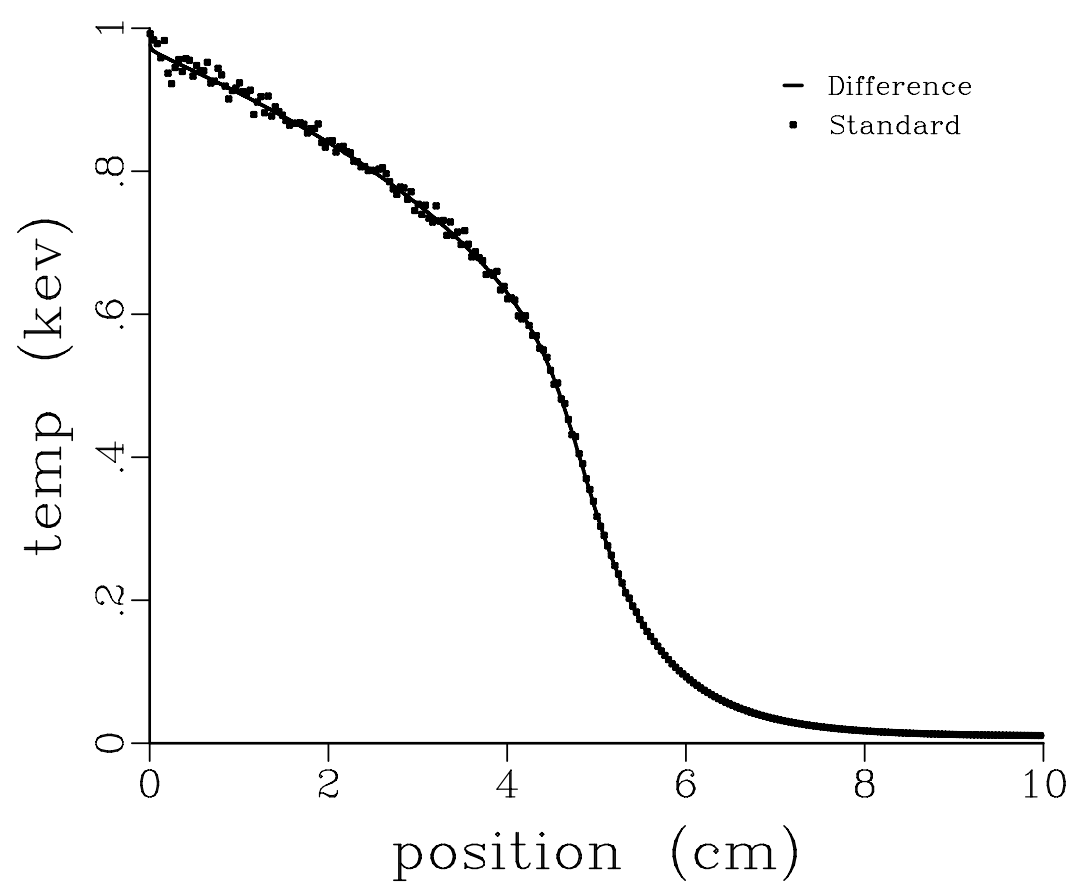

Fig. 6. Thermal wave penetrating a uniform slab, shown at a time $t=1 \mathrm{sh}$. The optical thickness of the slab below $1 \mathrm{keV}$ is 1000 mean free paths, but there is a precipitous drop in opacity by a factor of 100 above $1 \mathrm{keV}$. The long "foot" of the thermal wave stems from high frequency photons that penetrate deeply. 